\title{
Stand und Perspektiven der Chemotherapie beim anthrazyklinvorbehandelten Mammakarzinom - Ergebnisse randomisierter Studien
}

\author{
V. Heinemann \\ Med. Klinik III, Klinikum Großhadern, München
}

Schlüsselwörter

Fortgeschrittenes Mammakarzinom · Anthrazyklinvorbehandlung · Chemotherapie - Docetaxel · Taxane

\section{Zusammenfassung}

In randomisierten Studien zeigten Chemotherapien auf Taxanbasis beim anthrazyklinvorbehandelten Mammakarzinom eine gute Wirksamkeit. Im direkten Vergleich der Monosubstanzen erwies sich Docetaxel signifikant wirksamer als Paclitaxel, war allerdings auch mit höheren Grad-3/4-Toxizitäten verbunden. In zwei weiteren Studien waren Taxan-Kombinationen der Monotherapie deutlich überlegen. Erwartungsgemäß waren die Kombinationsregime toxischer, doch führte dies nicht zu einer Beeinträchtigung der Lebensqualität.

\section{Einleitung}

Die Chemotherapie gilt beim rezeptornegativen metastasierten Mammakarzinom als Behandlungsform der Wahl. Bei Patientinnen mit rezeptorpositiven Tumoren wird empfohlen, die Chemotherapie erst nach Versagen einer endokrinen Therapie oder bei Vorliegen von Symptomen und/oder einer massiven viszeralen Metastasierung einzusetzen.

Vor Einführung der Taxane waren Anthrazykline wie Doxorubicin oder Epirubicin infolge ihrer guten und schnellen Wirkung der Goldstandard für die Chemotherapie [1]. Mehrere Studien in der Prä-Taxan-Ära zeigten für Kombinationsregime auf Anthrazyklinbasis eine mäßige, aber konsistente Überlegenheit gegenüber einer Chemotherapie ohne Anthrazykline [2]. Aufgrund der Ergebnisse in der palliativen Thera-

\author{
Key Words \\ Advanced breast cancer . Anthracycline-pretreated . \\ Chemotherapy · Docetaxel - Taxanes
}

\section{Summary}

Current Status and Perspectives of Chemotherapy for Anthracycline-Pretreated Breast Cancer

Randomized trials performed in anthracycline-pretreated breast cancer patients have demonstrated good activity of taxane-based regimens. In a face to face comparison, single-agent docetaxel proved to be significantly more effective than paclitaxel. Treatment with docetaxel was also associated with an increased incidence of grade grade-3/4 toxicities in this trial. Antitumor activity of taxane-based combination regimens was clearly superior to single-agent therapy in two trials. As anticipated, treatment-associated toxicity was greater with combination regimens, but this did not affect quality of life.

pie wurden Anthrazykline dann auch in die adjuvante und neoadjuvante Therapie eingeführt, und sie gelten heute beim nodal positiven Mammakarzinom als Standardtherapie. Demzufolge ist in der klinischen Praxis bei Patientinnen mit fortgeschrittenem Mammakarzinom immer häufiger eine Anthrazyklinvorbehandlung anzutreffen.

Kommt es nach der First-line-Therapie zum Tumorprogress, ist die Chance einer Remission mit der Salvagetherapie meist geringer. Dies lässt darauf schließen, dass Zytostatikaresistenzen, die durch vorausgegangene Chemotherapien induziert wurden, über Kreuzresistenzmechanismen offenbar auch die nachfolgende Behandlung beeinträchtigen.

Zwar kommen Patientinnen mit Progress nach einer Anthrazyklinvorbehandlung immer noch für eine Chemotherapie in

\begin{tabular}{ll}
\hline KARGER & ( 2003 S. Karger GmbH, Freiburg \\
Fax +49 7614520714 & Accessible online at: \\
$\begin{array}{l}\text { E-mail Information@Karger.de } \\
\text { www.karger.com }\end{array}$ & www.karger.com/onk
\end{tabular}

PD Dr. med. Volker Heinemann 
Betracht, doch sprechen mehrere Argumente gegen eine Reexposition mit einem Anthrazyklin. Erstens besteht die Möglichkeit, dass der Tumor gegenüber der Anthrazyklinvorbehandlung refraktär war oder eine Zytostatikaresistenz induziert wurde. Zweitens schränkt die kumulative Kardiotoxizität der Anthrazykline eine wiederholte Behandlung ein [3], und drittens wird von vielen Patientinnen eine erneute Anthrazyklintherapie wegen ihrer früheren Erfahrungen mit Nebenwirkungen nicht akzeptiert.

Aus den genannten Gründen ist die Therapie anthrazyklinvorbehandelter Patientinnen zu einem Thema von zentralem klinischen Interesse geworden. Seine Bedeutung erkennt man daran, dass in randomisierten klinischen Studien lediglich 40-60\% der Patientinnen mit metastasiertem Mammakarzinom auf eine First-line-Therapie mit Anthrazyklinen ansprachen.

\section{Methoden}

Gegenstand der vorliegenden Übersichtsarbeit sind die Ergebnisse randomisierter Phase-III-Studien bei anthrazyklinvorbehandelten Patientinnen mit metastasiertem Mammakarzinom (Tab. 1, 2). Vollpublikationen wurden durch eine MEDLINE-Suche identifiziert. Darüber hinaus wurden Abstracts berücksichtigt, die auf den Tagungen der American Society of Clinical Oncology (ASCO) und der European Cancer Conference (ECCO) vorgestellt wurden

\section{Ergebnisse}

\section{Docetaxel-Monotherapie versus vs. Kombinationstherapie}

Docetaxel besitzt beim Mammakarzinom eine hohe Monoaktivität. Beim anthrazyklinresistenten metastasierten Mammakarzinom induziert es Remissionsraten von 30\% bis- $69 \%$ [4-8], so dass Docetaxel für diese Indikation die Zulassung als Monotherapie erhielt [9]. In drei randomisierten Phase-IIIStudien wurde eine Docetaxel-Monotherapie beim anthrazyklinvorbehandelten metastasierten Mammakarzinom mit Kombinationsregimen ohne Anthrazyklin und Taxan verglichen.

Die Scandinavian Breast Group [10] publizierte eine Vergleichsstudie von Docetaxel mit der Kombination Methotrexat und 5-Fluorouracil (MF); primärer Endpunkt war das progressionsfreie Intervall (TTP). Die Zahl der Patientinnen, die im Rahmen der adjuvanten Therapie ein Anthrazyklin erhalten hatten, war gering, ihr Anteil belief sich in den beiden Armen auf $17 \%$ bzw. $12 \%$. In dieser Studie ergab sich für Docetaxel eine signifikant bessere Ansprechrate (42 vs. 21\%) und TTP (6,3 vs. 3,0 Monate). Im Falle der Progression war ein Crossover zur jeweils anderen Therapie vorgesehen. Dies erfolgte bei $50 \%$ der Patientinnen und führte zu Ansprechraten von $27 \%$ mit Docetaxel und $12 \%$ mit MF. Während Docetaxel also in Bezug auf Remissionsrate und TTP die deutlich wirksamere Therapie war, kam ein möglicher Überlebens-vorteil im Docetaxel-Arm der Studie durch das Crossover-Design nicht zur Geltung (10,4 vs. 11,1 Monate).

Nabholtz und Mitarbeiter [11] verglichen Docetaxel als Monosubstanz mit der Kombination Mitomycin C plus Vinblastin (MV). Alle Patientinnen hatten zuvor ein Anthrazyklin erhalten, $26 \%$ bzw. 30\% von ihnen nur in der adjuvanten Situation. Zwar wird das Vergleichsregime MV beim Mammakarzinom kaum mehr eingesetzt; dennoch ist bemerkenswert, dass Docetaxel nicht nur einen signifikanten Vorteil bei Gesamtansprechrate (ORR) und dem primären Endpunkt TTP bot, sondern auch die mediane Überlebenszeit signifikant verlängerte (11,4 vs. 8,7 Monate).

In der neueren Phase-III-Studie von Bonneterre et al. [9] wurde die Wirksamkeit von Docetaxel mit der des Kombinationsregimes 5-Fluorouracil plus Vinorelbin (FUN) verglichen. $66 \%$ aller Patientinnen hatten im fortgeschrittenen Stadium bereits eine Chemotherapie erhalten und 36\% bzw. $43 \%$ wurden in den beiden Studienarmen als anthrazyklinrefraktär oder -resistent eingestuft. Im Gegensatz zur Studie von Nabholtz et al. erwiesen sich die Monotherapie mit Docetaxel und das FUN-Regime bezüglich ORR, TTP und Gesamtüberleben (OS) als gleichwertig. Dies galt auch für das Teilkollektiv von anthrazyklinrefraktären bzw. -resistenten Patientinnen: ORR 39 vs. $23 \%$, TTP 6,2 vs. 4,3 Monate und OS 11,5 vs. 11,5 Monate. In einer multivariaten Analyse korrelierten die Anthrazyklinsensitivität und die Zahl metastasenbefallener Organe mit dem progressionsfreien Intervall. Die Patientinnen der Bonneterre-Studie unterschieden sich von denen der Nabholtz-Studie [11] darin, dass bei letzteren eine Anthrazyklinresistenz häufiger (56 vs. $40 \%$ ) und das prognostische Profil ungünstiger war. Entsprechend waren im Docetaxel-Arm der Bonneterre-Studie die Remissionsrate höher sowie TTP und Überleben länger [9].

Insgesamt belegen diese Studien, dass Docetaxel ein Zytostatikum mit konsistent hoher Aktivität beim anthrazyklinvorbehandelten Mammakarzinom ist. Kein anderer Wirkstoff wurde in dieser speziellen Krankheitssituation eingehender in randomisierten Studien geprüft. Im Vergleich mit verschiedenen Kombinationsregimen erwies sich die Monotherapie mit Docetaxel überlegen oder gleichwertig. Die Ergebnisse der skandinavischen Studie deuten darauf hin, dass es für die Überlebenszeit unerheblich ist, in welcher Therapielinie (1stoder 2nd-line) Docetaxel eingesetzt wird, sofern die Behandlung unmittelbar nach Eintritt der Tumorprogression aufgenommen wird [10].

\section{Der nächste Schritt: Docetaxel in Kombination mit Capecitabin}

Nach Etablierung der Docetaxel-Monotherapie in der Salvagetherapie anthrazyklinvorbehandelter Patientinnen stellte sich die Frage, ob nicht Kombinationsregime mit Docetaxel mögli- 
Tab. 1. Randomisierte Studien beim anthrazyklinvorbehandelten Mammakarzinom - Wirksamkeit

\begin{tabular}{|c|c|c|c|c|c|c|c|c|c|}
\hline Autoren & $\begin{array}{l}\text { Schema } \\
\text { (Abk.) }\end{array}$ & Schema (Dosierung in $\mathrm{mg} / \mathrm{m}^{2}$ ) & $\begin{array}{l}\text { Patien- } \\
\text { ten, } \mathrm{n}\end{array}$ & $\begin{array}{l}\text { ORR, } \\
\%\end{array}$ & $\mathrm{p}$ & $\begin{array}{l}\text { TTP, } \\
\text { Monate }\end{array}$ & $\mathrm{p}$ & $\begin{array}{l}\text { Überleben } \\
\text { (Monate) }\end{array}$ & $\mathrm{p}$ \\
\hline Sjöström et al. [10] & $\begin{array}{l}\text { Doc } \\
\text { MF }\end{array}$ & $\begin{array}{l}\text { Docetaxel 100, q } 3 \text { wks } \\
\text { MTX 200, 5-FU } 600 \text { d 1+8, q } 3 \text { wks }\end{array}$ & $\begin{array}{l}143 \\
139\end{array}$ & $\begin{array}{l}42 \\
21\end{array}$ & $<0,001$ & $\begin{array}{l}6,3 \\
3,0\end{array}$ & $<0,001$ & $\begin{array}{l}10,4 \\
11,1\end{array}$ & n.s. \\
\hline Nabholtz et al. [12] & $\begin{array}{l}\text { Doc } \\
\text { MV }\end{array}$ & $\begin{array}{l}\text { Docetaxel 100, q } 3 \text { wks } \\
\text { Mitomycin } 12 \mathrm{mg} \text {, q } 6 \text { wks, VBL } 6 \text { mg q } 3 \text { wks }\end{array}$ & $\begin{array}{l}203 \\
189\end{array}$ & $\begin{array}{l}30 \\
11,6\end{array}$ & $<0,0001$ & $\begin{array}{l}4,8 \\
2,8\end{array}$ & 0,001 & $\begin{array}{r}11,4 \\
8,7\end{array}$ & 0,0097 \\
\hline Bonneterre et al. [9] & $\begin{array}{l}\text { Doc } \\
\text { FUN }\end{array}$ & $\begin{array}{l}\text { Docetaxel 100, q } 3 \text { wks } \\
5 \text {-FU } 750 \text { CI, d1-5; Vinorelbin } 25 \text { d 1+5, q 3w }\end{array}$ & $\begin{array}{l}86 \\
90\end{array}$ & $\begin{array}{l}43 \\
39\end{array}$ & n.s. & $\begin{array}{l}6,5 \\
5,1\end{array}$ & n.s. & $\begin{array}{l}16 \\
15\end{array}$ & n.s. \\
\hline $\begin{array}{l}\text { O'Shaughnessy et al. } \\
2002[12]\end{array}$ & $\begin{array}{l}\text { Doc } \\
\text { DocCap }\end{array}$ & $\begin{array}{l}\text { Docetaxel 100, q } 3 \text { wks } \\
\text { Doc } 75 \text {; Capecitabin } 2500 \text { po, d 1-14, q } 3 \text { wks }\end{array}$ & $\begin{array}{l}256 \\
255\end{array}$ & $\begin{array}{l}30 \\
42\end{array}$ & 0,006 & $\begin{array}{l}4,2 \\
6,1\end{array}$ & 0,0001 & $\begin{array}{l}11,5 \\
14,5\end{array}$ & 0,0126 \\
\hline Ravdin [20] & $\begin{array}{l}\text { Doc } \\
\text { Pac }\end{array}$ & $\begin{array}{l}\text { Docetaxel 100, q } 3 \text { wks } \\
\text { Paclitaxel 175, q } 3 \text { wks }\end{array}$ & $\begin{array}{l}225 \\
224\end{array}$ & $\begin{array}{l}32 \\
25\end{array}$ & n.s. & $\begin{array}{l}5,7 \\
3,6\end{array}$ & 0,0001 & $\begin{array}{l}15,4 \\
12,7\end{array}$ & 0,03 \\
\hline Dieras et al. [17] & $\begin{array}{l}\text { Pac } \\
\text { Mito }\end{array}$ & $\begin{array}{l}\text { Paclitaxel 175, q } 3 \text { wks } \\
\text { Mitomycin } 12 \text { mg, q } 6 \text { wks }\end{array}$ & 81 & $\begin{array}{r}17 \\
6\end{array}$ & n.s. & $\begin{array}{l}3,5 \\
1,6\end{array}$ & 0,026 & n.a. & n.a. \\
\hline $\begin{array}{l}\text { O'Shaughnessy et al. } \\
2003 \text { [19] }\end{array}$ & $\begin{array}{l}\text { Pac } \\
\text { PacGem }\end{array}$ & $\begin{array}{l}\text { Paclitaxel 175, q } 3 \text { wks } \\
\text { Gemcitabin } 1250 \text { d } 1+8 \text {; Pac } 175 \text { d 1, q } 3 \text { wks }\end{array}$ & 529 & $\begin{array}{l}26 \\
39\end{array}$ & 0,0007 & $\begin{array}{l}3,5 \\
5,4\end{array}$ & 0,0013 & n.a. & n.a. \\
\hline Jones et al. [21] & $\begin{array}{l}\text { VNR } \\
\text { Mel }\end{array}$ & $\begin{array}{l}\text { Vinorelbin } 30 \text { wöchentlich } \\
\text { Melphalan } 25 \text {, q } 4 \text { wks }\end{array}$ & $\begin{array}{r}115 \\
64\end{array}$ & $\begin{array}{r}16 \\
9\end{array}$ & n.s. & $\begin{array}{l}3 \\
2\end{array}$ & 0,001 & $\begin{array}{l}8,8 \\
7,8\end{array}$ & 0,034 \\
\hline
\end{tabular}

5-FU = 5-Fluorouracil; CI = kontinuierliche Infusion; Doc = Docetaxel; MTX = Methotrexat; n.a. = nicht angegeben; n.s. = nicht signifikant; ORR $=$ Gesamtansprechrate; Pac $=$ Paclitaxel; TTP $=$ progressionsfreies Intervall; VBL $=$ Vinblastin.

aphase-II-Studie.

cherweise noch besser wirksam sind. Aus verschiedenen Gründen wurde Capecitabin als Kombinationspartner ausgewählt: In mehreren Phase-II-Studien wurden mit dieser Substanz Ansprechraten von $15-26 \%$ bei Patientinnen erreicht, die zuvor ein Anthrazyklin und Taxan erhalten hatten [12]. Capecitabin ist auch aufgrund seiner geringen myelosuppressiven Wirkung besonders für die Kombinationstherapie geeignet. Ferner wurde in präklinischen Untersuchungen nachgewiesen, dass die Taxanexposition zu einer Hochregulierung der Thymidinphosphorylase führt, also jenes Enzyms, das für die intrazelluläre Aktivierung von Capecitabin verantwortlich ist. In Bestätigung dieser molekularen Wechselwirkung ergab sich in Xenograft-Modellen bei gleichzeitiger Anwendung von Capecitabin und Taxanen ein Wirkungssynergismus.

Aufgrund dieser präklinischen Ergebnisse verglichen O'Shaughnessy et al. [13] in einer groß angelegten randomisierten Studie an 511 Patientinnen das Kombinationsregime Docetaxel/Capecitabin (DX) mit Docetaxel alleine. Alle Patientinnen waren mit einem Anthrazyklin vorbehandelt, wobei $60 \%$ (DX) bzw. 64\% (Docetaxel) das Anthrazyklin im metastasierten Stadium erhalten hatten. Die Kombinationstherapie mit DX induzierte nicht nur eine signifikant höhere Remissionsrate (42 vs. $30 \%)$, sondern sie verlängerte auch signifikant das progressionsfreie Intervall (6,1 vs. 4,2 Monate) und vor allem die mediane Überlebenszeit (14,5 vs, 11,5 Monate). Die der
Studientherapie folgende Behandlung war in den beiden Gruppen recht ähnlich, wenngleich im Kombinationsarm häufiger Docetaxel (20 vs. 7\%) und im Docetaxel-Arm häufiger Capecitabin (17 vs. 3\%) als Folgetherapie eingesetzt wurde. Die umfassende Beurteilung des allgemeinen Gesundheitszustandes (global health status) ergab tendenziell bessere Werte im Kombinationsarm; dieser Umstand verdeutlicht, dass der Überlebensvorteil mit der Kombinationstherapie nicht zu Lasten der Lebensqualität ging. Zudem unterstrich eine pharmakoökonomische Analyse, dass die Kombination Docetaxel/Capecitabin eine kosteneffiziente Therapie darstellt; die durch Capecitabin entstehenden Mehrkosten wurden durch die geringere Docetaxeldosis nahezu ausgeglichen [12].

\section{Therapieregime mit Paclitaxel}

Aufschluss über die Wirksamkeit von Paclitaxel beim anthrazyklinvorbehandelten metastasierten Mammakarzinom geben mehrere randomisierte Studien. In einer Studie der EORTC [14] wurde in der First-line-Situation Paclitaxel mit Doxorubicin (jeweils als Monotherapie) verglichen. Bei Tumorprogress wurde auf die jeweils andere Therapie umgestellt. Von den 77 Patientinnen, die Paclitaxel im Anschluss an Doxorubicin erhielten, sprachen nur $16 \%$ an, dagegen betrug die Ansprech- 
Tab. 2. Randomisierte Studien beim anthrazyklinvorbehandelten Mammakarzinom - Toxizität

\begin{tabular}{|c|c|c|c|c|c|}
\hline Autoren & $\begin{array}{l}\text { Schema } \\
\text { (Abkürzung) }\end{array}$ & Patienten, n & $\begin{array}{l}\text { Neutropenie } \\
\text { Grad 3-4, \% }\end{array}$ & Febrile Neutropenie, \% & $\begin{array}{l}\text { Therapiebedingte } \\
\text { Mortalität, \% }\end{array}$ \\
\hline \multirow[t]{2}{*}{ Sjöström et al. [10] } & Doc & 143 & $77^{\mathrm{a}}$ & \multirow{2}{*}{ n.a. } & 2,1 \\
\hline & MF & 139 & $16^{\mathrm{a}}$ & & 0,7 \\
\hline \multirow[t]{2}{*}{ Nabholtz et al. [11] } & Doc & 203 & 93,1 & 9 & 2,0 \\
\hline & MV & 189 & 62,5 & 0,5 & 1,6 \\
\hline \multirow[t]{2}{*}{ Bonneterre et al. [9] } & Doc & 86 & 82 & \multirow{2}{*}{ n.a. } & 1,2 \\
\hline & FUN & 90 & 67 & & 5,6 \\
\hline \multirow[t]{2}{*}{ O'Shaughnessy et al. 2002 [12] } & Doc & 256 & 15 & 21 & 0,4 \\
\hline & DocCap & 255 & 16 & 16 & 1,2 \\
\hline \multirow[t]{2}{*}{ Ravdin [20] } & Doc & 225 & 93,3 & 14,4 & \multirow{2}{*}{ n.a. } \\
\hline & $\mathrm{Pac}$ & 224 & 54,5 & 3,4 & \\
\hline \multirow[t]{2}{*}{ O’Shaughnessy et al. 2003 [19] } & $\mathrm{Pac}$ & \multirow{2}{*}{529} & 11 & 2 & $<1$ \\
\hline & PacGem & & 48 & 5 & $<1$ \\
\hline \multirow[t]{2}{*}{ Jones et al. [21] } & VNR & 115 & 75 & $10^{\mathrm{b}}$ & 0 \\
\hline & Mel & 64 & 69 & $8^{b}$ & 0 \\
\hline
\end{tabular}

n.a. = Nicht angegeben.

${ }^{\mathrm{a}} \mathrm{Als}$ Leukopenie angegeben.

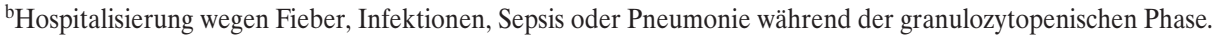

rate $30 \%$ bei den 91 Patientinnen, die nach First-line-Therapie mit Paclitaxel auf Doxorubicin umgestellt wurden. Die mediane Überlebenszeit war mit First-line-Doxorubicin länger (allerdings nicht signifikant) als mit Paclitaxel (18,3 vs. 15,6 Monate).

An der kürzlich veröffentlichten dreiarmigen Intergroup-Studie von Sledge et al. [15] nahmen 739 Patientinnen teil. Sie erhielten als First-line-Therapie entweder eine Monotherapie mit Paclitaxel oder Doxorubicin oder eine Kombinationstherapie mit Doxorubicin plus Paclitaxel. Nach Eintreten der Tumorprogression wurde in den Monotherapiearmen auf das jeweils andere Zytostatikum umgestellt. Nach Anthrazyklinvorbehandlung betrug die Ansprechrate mit Paclitaxel in der Second-line-Situation 20\%, mit Doxorubicin nach Paclitaxelvorbehandlung $22 \%$. Als wichtigste Erkenntnis ergab sich aus dieser Studie, dass beide Therapiesequenzen (Doxorubicin $\rightarrow$ Paclitaxel und Paclitaxel $\rightarrow$ Doxorubicin) und die Kombinationstherapie mit beiden Substanzen zu einer fast gleichen medianen Überlebenszeit führten (18,9 vs. 22,2 vs. 22,0 Monate). In einer weiteren Studie wurde die Wirksamkeit einer Chemotherapie mit oder ohne Trastuzumab bei Patientinnen mit Überexpression von HER-2/neu untersucht [16]. Bei den 96 Patientinnen, die im Rahmen ihrer adjuvanten Therapie Anthrazykline erhalten hatten, betrug die Ansprechrate mit $\mathrm{Pa}$ clitaxel $16 \%$.

Daten zur relativen Wirksamkeit von Paclitaxel als Monosubstanz liegen aus einer kleinen randomisierten Phase-IIStudie ( $\mathrm{n}=81$ ) vor, in der Paclitaxel mit Mitomycin verglichen wurde [17]. Mit einer einzigen Ausnahme waren alle Patientinnen mit einem Anthrazyklin vorbehandelt. Während sich die Ansprechraten mit Paclitaxel und Mitomycin nicht signifikant voneinander unterschieden ( 17 vs. $6 \%, p=0,14)$, ergab sich mit Paclitaxel ein signifikant längeres progressionsfreies Intervall (3,5 vs. 1,6 Monate, $\mathrm{p}=0,026)$.

Insgesamt wurden mit Paclitaxel in randomisierten Studie beim anthrazyklinvorbehandelten Mammakarzinom recht einheitliche Remissionsraten von 16-20\% erzielt. Aus zwei Studien geht hervor, dass die Reihenfolge einer Monotherapie mit Doxorubicin und Paclitaxel ohne signifikanten Einfluss auf das Überleben ist.

\section{Gemcitabin/Paclitaxel vs. Paclitaxel}

Der kombinierten Therapie mit Gemcitabin und Paclitaxel liegt die Überlegung zugrunde, dass beide Substanzen einen unterschiedlichen Wirkungsmechanismus besitzen und sich ihr Toxizitätsspektrum nur wenig überlappt. Gemcitabin besitzt eine bemerkenswert geringe Toxizität, wobei die Myelosuppression dosislimitierend ist. In Phase-II-Studien induzierte die Kombination Gemcitabin/Paclitaxel Ansprechraten von 55-68\% [18]. Auf der ASCO-Tagung 2003 präsentierten O'Shaughnessy et al. [19] die Zwischenergebnisse einer Phase-III-Studie, in der die Kombination Gemcitabin/ Paclitaxel mit einer Paclitaxel-Monotherapie verglichen wurde. An dieser Studie nahmen 529 anthrazyklinvorbehandelte Patientinnen teil. Im Gegensatz zu vorausgegangenen Studien hatten $96 \%$ der Patientinnen das Anthrazyklin in der adjuvanten Situation erhalten. Die Gesamtansprechrate im Kombinationarm war signifikant höher als im Paclitaxel-Arm (39,3 vs. $25,6 \%, p=0,0007)$; ebenso war das progressionsfreie Intervall mit der Kombination signifikant länger (5,4 vs. 3,5 Monate, $p$ $=0,0013)$. Überlebensdaten für die beiden Studienarme wurden noch nicht vorgelegt. 
Vergleich von Docetaxel und Paclitaxel

Die erste größere randomisierte Vergleichsstudie $(n=449)$ zwischen Docetaxel und Paclitaxel wurde von Ravdin et al. [20] durchgeführt. Die Einschlussbedingungen erlaubten maximal eine Vorbehandlung mit einem anthrazyklinhaltigen Regime und schlossen eine Taxanvorbehandlung aus. Die Therapiemedikation wurde fortgesetzt bis zur inakzeptablen Toxizität oder dem Fortschritt der Erkrankung (Progression). Für die meisten Patientinnen war die Studientherapie die Zweitlinientherapie im metastasierten bzw. lokal fortgeschrittenen Stadium ihres Mammakarzinoms. Bei der Berechnung der Gesamtansprechrate auf Intention-to-treat-Basis war der Unterschied zwischen Docetaxel und Paclitaxel nicht signifikant (32 vs. 25\%); gingen jedoch nur die auswertbaren Patientinnen in die Berechnung ein, war die Ansprechrate mit Docetaxel signifikant höher $(37,4$ vs. $26,4 \%, p=0,02)$. Dies schlug sich auch in der ITT-Population in einem signifikant besseren progressionsfreien Intervall (5,7 vs. 3,6 Monate, $\mathrm{p}=$ $0,0001)$ und einer signifikant längeren Überlebenszeit (15,4 vs. 12,7 Monate, $\mathrm{p}=0,03$ ) in der Docetaxelgruppe nieder. Febrile Neutropenien (15 vs. $2 \%, \mathrm{p}<0,05)$ und Infektionen Grad 3-4 $(10$ vs. $2 \%, p<0,05)$ waren unter Docetaxel häufiger. Gleiches galt für die meisten anderen einschließlich neuromotorischer Nebenwirkungen. Jedoch muss hierbei die höhere mediane Zyklenzahl unter Docetaxel berücksichtigt werden (6 vs. 4).

\section{Vinorelbin}

Jones et al. [21] verglichen an 183 anthrazyklinvorbehandelten Patientinnen die klinische Wirkung von Vinorelbin mit der von Melphalan; die Randomisierung erfolgte im Zahlenverhältnis 2:1. Nur 9\% der Patientinnen im Vinorelbin-Arm und 8\% im Melphalan-Arm dieser Studie hatten das Anthrazyklin in der adjuvanten Situation erhalten. Die Gesamtansprechrate war in beiden Studienarmen vergleichsweise gering (16\% mit Vinorelbin vs. $9 \%$ mit Melphalan). Beim progressionsfreien Intervall ergab sich ein kleiner, aber signifikanter Vorteil zugunsten von Vinorelbin ( 3 vs. 2 Monate, $\mathrm{p}=0,0001$ ), der in diesem Studienarm auch zu einem etwas längeren Überleben führte (8,75 vs. 7,75 Monate, $\mathrm{p}=0,034)$. Im Vergleich mit den Taxanen hat Vinorelbin zwar das eindeutig günstigere Toxizitätsprofil, doch die wenigen Daten, die bislang von anthrazyklinvorbehandelten Patientinnen vorliegen, deuten darauf hin, dass es auch weniger wirksam ist als Docetaxel.

\section{Diskussion}

Anhand einer Gegenüberstellung randomisierter Studien lässt sich zwar ein Überblick über die Datenlage und deren Evidenz gewinnen, jedoch ist beim Vergleich von Ergebnissen über verschiedene Studien hinweg grundsätzlich Vorsicht geboten. Auf diese Weise lassen sich zwar Hypothesen generieren, aber keine gesicherten Schlüsse ziehen. Das liegt u. a. daran, dass sich die Einschlusskriterien von Studien teilweise deutlich voneinander unterscheiden. So lag der Anteil von Patientinnen, die mit einer ausschließlich adjuvanten Anthrazyklinvorbehandlung in die oben besprochenen Studien aufgenommen wurden, zwischen 12 und $96 \%$. Außerdem wurden Anthrazyklinresistenz und -refraktärität in den Studien teilweise unterschiedlich definiert. Eindeutige Aussagen zur relativen Wirksamkeit verschiedener Zytostatika lassen sich daher nur im direkten Vergleich in ein und derselben Studie gewinnen.

Zwar besteht in den meisten Studien ein Zusammenhang zwischen Gesamtansprechrate und progressionsfreiem Intervall, doch lässt sich aus diesen Parametern nicht unbedingt auf die Überlebenszeit rückschließen. Bei der Interpretation von Studienergebnissen sollte berücksichtigt werden, dass die Überlebensprognose nicht nur von der therapeutischen Intervention, sondern u. a. auch von der Tumorbiologie und der Wahl der Folgetherapie(n) abhängt. Die Anschlussbehandlung kann zwischen verschiedenen Studien sehr variieren und wird zumeist nicht vom Studienprotokoll vorgeschrieben. Interessanterweise erwies sich die Reihenfolge der Verabreichung von Anthrazyklinen und Taxanen ohne Einfluss auf das Überleben, wenn für beide Substanzen ein Crossover vorgesehen war $[14,15]$.

Lange Zeit existierte für das anthrazyklinvorbehandelte Mammakarzinom keine Standardchemotherapie. Mit zunehmender Zahl randomisierter Studien versteht man aber immer besser, wie eine adäquate Therapie in dieser Situation aussehen könnte. Dennoch gibt es nach wie vor widersprüchliche Ansichten. Zunächst ist immer noch unklar, welche $\mathrm{Pa}-$ tientinnen als anthrazyklinsensitiv oder -resistent einzustufen sind, wenn es nach einer adjuvanten Anthrazyklinbehandlung zu einem Rezidiv kommt. Man geht davon aus, dass eine Anthrazyklinsensitivität umso wahrscheinlicher anzunehmen ist, je länger das rezidivfreie Intervall war. Demnach könnten Patienten mit einem Spätrezidiv (z.B. mehr als 12 Monate nach Abschluss der adjuvanten Anthrazyklintherapie) eventuell für eine erneute Behandlung mit einem Anthrazyklin in Frage kommen. Um eine kumulative Kardiotoxizität zu vermeiden, ist in dieser Situation am ehesten liposomalem Doxorubicin der Vorzug zu geben.

Es hat sich gezeigt, dass zwischen den Taxanen Docetaxel oder Paclitaxel und den Anthrazyklinen nur eine unvollständige Kreuzresistenz besteht. In drei Studien beim anthrazyklinvorbehandelten Mammakarzinom erwies sich die Monotherapie mit Docetaxel verschiedenen Kombinationsregimen (Methotrexat + 5-Fluorouracil, Mitomycin C + Vinblastin, 5Fluorouracil + Vinorelbin) überlegen (2 Studien) bzw. ebenbürtig (1 Studie) [9-11]. Aufgrund dieser Studien gilt Docetaxel als Standardtherapie, mit der eine hohe Remissionsrate von $30-43 \%$ erwartet werden kann.

Die Phase-III-Datenlage zu Paclitaxel beim anthrazyklinvorbehandelten Mammakarzinom ist weniger umfangreich. Aus 
drei Phase-III-Studien liegen lediglich Subgruppenanalysen vor, die reproduzierbare Ansprechraten zwischen 16\% und 20\% ausweisen [14-16]. Obwohl die Konsistenz der Daten auf eine überlegene Wirksamkeit von Docetaxel bei anthrazyklinvorbehandelten Patientinnen hindeutete, ließen die vorhandenen Ergebnisse bislang keine eindeutigen Schlüsse zu. Diese Ungewissheit scheint nun durch den direkten Vergleich der beiden Taxane in einer randomisierten Studie ausgeräumt, denn hier ergab sich ein signifikanter Wirkungsvorteil von Docetaxel [20].

Für die Monotherapie beim anthrazyklinvorbehandelten Mammakarzinom kann Docetaxel nun als Zytostatikum der Wahl angesehen werden. Das gilt insbesondere für Patientinnen mit einem guten Allgemeinzustand, die eine Neutropenie Grad 3-4 tolerieren können (Tab. 2). Für Patientinnen in weniger guter körperlicher Verfassung und solche mit eingeschränkter Knochenmarkreserve ist eher eine Behandlung mit Paclitaxel oder Vinorelbin in Betracht zu ziehen, u.U. auch Docetaxel in reduzierter (bisher nicht in vergleichenden Studien geprüften) Dosierung. Die Monoaktivität neuerer Substanzen wie Capecitabin oder Gemcitabin wurde bislang nur in Phase-II-Studien dokumentiert. Beide Zytostatika erwie- sen sich beim vorbehandelten Mammakarzinom als wirksam und gut verträglich, vergleichende Daten aus Phase-III-Studien stehen aber noch aus [12,18].

Nachdem man die optimale Monotherapie für anthrazyklinvorbehandelte Patientinnen gefunden hatte, war es ein nächster logischer Schritt, die Wirksamkeit von Kombinationsregimen zu untersuchen. In einer randomisierten Studie erwies sich die Kombination Docetaxel/Capecitabin in Bezug auf Ansprechrate, progressionsfreies Intervall und Überleben signifikant wirksamer als die Monotherapie mit Docetaxel [13]. Wiederum traten im Kombinationsarm mehr Nebenwirkungen Grad 3 auf (71 vs. 49\%), so dass diese Therapie vor allem Patientinnen mit symptomatischer Erkrankung oder mit massiver viszeraler Metastasierung vorbehalten bleibt (Tab. 2).

Für die Kombination Paclitaxel/Gemcitabin wurde ebenfalls ein signifikanter Wirkungsvorteil gegenüber der Monotherapie mit Paclitaxel nachgewiesen, bislang allerdings nur für Ansprechrate und progressionsfreies Intervall. Eine endgültige Bewertung ist daher erst möglich, wenn solide Überlebensdaten vorliegen. Auch in dieser Studie war die Kombinationstherapie mit einer höheren hämatologischen Toxizität verbunden (Neutropenie Grad 3-4 48 vs. 11\% mit der Monotherapie).

\section{Literatur}

1 Valero V, Hortobagyi GN: (Editorial). Are anthracycline-taxane regimens the new standard of care in the treatment of metastatic breast cancer (Editorial)? J Clin Oncol 2003;21:959-962.

2 Fossati R, Confalonieri C, Torri V, Ghislandi E, Penna A, Pistotti V, Tinazzi A, Liberati A. : Cytotoxic and hormonal treatment for metastatic breast cancer: a systematic review of published randomized trials involving 31,510 women. J Clin Oncol 1998;16: 34-39.

3 Swain SM, Whaley FS, Ewer MS. : Congestive heart failure in patients treated with doxorubicin - a retrospective analysis of three trials. Cancer 2003;97:28692879 .

4 Ravdin PM, Burris III HA, Cook G, Eisenberg O, Kanes M, Bierman WA, Mortimer J, Genevois E, Bellet RE. : Phase II trial of docetaxel in advanced anthracycline-resistant or anthracenedione-resistant breast cancer. J Clin Oncol 1995;13:2879-2885.

5 Valero V, Holmes FA, Walters RS, Theriault RL, Esparza L, Fraschini G, Fonseca GA, Bellet RE, Buzdar AU, Hortobagyi GN. : Phase II trial of docetaxel: a new highly effective antineoplastic agent in the management of patients with anthracycline-resistant metastatic breast cancer. J Clin Oncol 1995;13:28862894.

6 Nabholtz JM, Crown J. : Phase III studies of singleagent docetaxel in patients with metastatic breast cancer who have progressed despite previous chemotherapy regimens: preliminary results. Semin Oncol 1998;25:4-9.

7 Alexopoulos CG, Rigatos G, Efremidis AP, Papacharalambous A, Alexopoulos A, Vassilomanolakis M, Patila E. : A phase II study of the effectiveness of docetaxel (Taxotere) in women with advanced breast cancer previously treated with polychemotherapy. Helleneic Cooperative Interhospital Group in Oncology. Cancer Chemother Pharmacol 1999;44:253-258.

8 Brodowicz T, Koestler WJ, Tomek S, Vaclavik I, Herscovici V, Wiltschke C, Steger GG; Zielinski CC. Monotherapy with docetaxel in second- or thirdline treatment of anthracycline-resistant metastatic breast cancer. Anticancer Drugs 2000;11:149-153.
9 Bonneterre J, Roché H, Monnier A, Guastalle JP, Namer M, Fargeot P, Assadourian S. : Docetaxel vs 5 -fluorouracil plus vinorelbine in metastatic breast cancer after anthracycline therapy failure. $\mathrm{Br} \mathrm{J}$ Cancer 2002;87:1210-1215.

10 Sjöström J, Blomqvist C, Mouridsen H, Pluzanska A, Ottosson-Lönn S, Bengtsson N-O, Ostenstad B, Mjaaland I, Palm-Sjövall M, Wist E, Valvere V, Anderson H, Bergh J. : Docetaxel compared with sequential methotrexate and 5-fluorouracil in patients with advanced breast cancer after anthracycline failure: a randomised phase III study with crossover on progression by the Scandinavian Breast Group. Eur J Cancer 1999;35:1194-1201.

11 Nabholtz J-M, Senn HJ, Bezwoda WR, Melnychuk D, Deschênes L, Douma J, Vandenberg TA, Rapoport B, Rosso R, Trillet-Lenoir V, Drbal J, Molino A, Nortier JWR, Richel DJ, Nagykalnai T, Siedlecki P, Wilking N, Genot JY, Hupperets PSGJ, Pannuti F, Skarlos D, Tomiak EM, Murawsky M, Alaki M, Riva A, Aapro M. : Prospective randomized trial of docetaxel versus mitomycin plus vinblastine in patients with metastatic breast cancer progressing despite previous anthracycline-containing chemotherapy. J Clin Oncol 1999;17:1413-1424.

12 O'Shaughnessy J, Twelves C, Aapro M. Treatment for anthracycline-pretreated metastatic breast cancer. Oncologist 2002;7(suppl 6):4-12.

13 O'Shaughnessy J, Miles D, Vukelja S, Moiseyenko V, Ayoub J-P, Cervantes G, Fumoleau P, Jones S, Lui WY, Mauriac L, Twelves C, Van Hazel G, Verma S, Leonard R. Superior survival with capecitabine plus docetaxel combination therapy in anthracycline-pretreated patients with advanced breast cancer: Phase III trial results. J Clin Oncol 2002;20:2812-2823.

14 Paridaens R, Biganzoli L, Bruning P, Klijn JGM, Gamucci T, Houston S, Coleman R, Schachter J, Van Vreckem A, Sylvester R, Awada A, Wildiers J, Piccart M. : Paclitaxel versus doxorubicin as first-line single agent chemotherapy for metastatic breast cancer: A European Organization for Research and Treatment of Cancer randomized study with crossover. J Clin Oncol 2000;18:724-733.
15 Sledge GW, Neuberg D, Bernardo P, Ingle JN, Martino S, Rowinsky EK, Wood WC: Phase III trial of doxorubicin, paclitaxel, and the combination of doxorubicin and paclitaxel as front-line chemotherapy for metastatic breast cancer: An Intergroup trial (E1193). J Clin Oncol 2003;21:588-592.

16 Slamon DJ, Leyland-Jones B, Shak S, Fuchs H, Paton V, Bajamonde A, Fleming T, Eiermann W, Wolter J, Pegram M, Baselga J, Norton L. : Use of chemotherapy plus monoclonal antibody against HER2 for metastatic breast cancer that overexpresses HER2. N Engl J Med 2001;344:783-792.

17 Dieras V, Marty M, Tubiana N, Corette L, Morvan F, Serin D, Mignon, Chazard M, Garet F, Onetto N, Hellmann S, Pouillart P. : Phase II randomized study of paclitaxel versus mitomycin in advanced breast cancer. Semin Oncol 1995;22(Suppl 8):33-39.

18 Heinemann V. : Role of gemcitabine in the treatment of advanced and metastatic breast cancer. Oncology 2003;64:191-206

19 O'Shaughnessy J, Nag S, Calerillo-Ruiz G, Jordaan J, Llombart A, Pluzanska A, Pawlicki M, Reyes JM, Sekhon J, Albain KS. : Gemcitabine plus paclitaxel (GT) versus paclitaxel (T) as first-line treatment for anthracycline pre-treated metastatic breast cancer (MBC): Interim results of a global phase III study. Proc Am Soc Clin Oncol 2003;22:7(Abstr abstr 25).

20 Ravdin P. : Phase III comparison of docetaxel (D) and paclitaxel $(\mathrm{P})$ in patients with metastatic breast cancer (MBC). Abstract presented at ECCO 2003, Copenhagen.

21 Jones S, Vogel C, Laufman L, Hutchins L, O’Rourke M, Lembersky B, Budman D, Bigley J, Hohneker J. Randomized comparison of vinorelbine and melphalan in anthracycline-refractory advanced breast cancer. J Clin Oncol 1995;13:2567-2574. 